Int. J. Electrochem. Sci., 11 (2016) 9295 - 9306

\title{
Characterization of Zeolitic Imidazolate Framework-derived Polyhedral Carbonaceous Material and its Application to Electrocatalyst for Oxygen Reduction Reaction
}

\author{
Kyo Sung Park ${ }^{1, \dagger}$, Seon-ah Jin ${ }^{1, \dagger}$, Kang Hee Lee ${ }^{1, *}$, Junho Lee $^{2}$, Inyong Song ${ }^{2}$, Byoung-Sun Lee ${ }^{2}$, \\ Sookyung Kim ${ }^{3}$, Jeongsoo Sohn ${ }^{3}$, Chanho Pak ${ }^{4}$, Gunha Kim ${ }^{5}$, Seok-Gwang Doo ${ }^{1}$, Kyungjung Kwon ${ }^{5, *}$ \\ ${ }^{1}$ Energy Material Laboratory, and ${ }^{2}$ Analytical Engineering Group, Samsung Advanced Institute of \\ Technology, 130 Samsung-ro, Yeongtong-gu, Suwon, Gyeonggi-do, Korea \\ ${ }^{3}$ Urban Mine Department, Korea Institute of Geoscience and Mineral Resources, 124 Gwahang-no, \\ Yuseong-gu, Daejeon, Korea \\ ${ }^{4}$ Institute of Integrated Technology, Gwangju Institute of Science and Technology, Gwangju, Korea \\ ${ }^{5}$ Department of Energy \& Mineral Resources Engineering, Sejong University, Seoul, Korea \\ *E-mail: kanghee.lee@ samsung.com, kfromberk@gmail.com \\ $\dagger$ These authors contributed equally to this work.
}

doi: $10.20964 / 2016.11 .27$

Received: 1 July 2016 / Accepted: 26 August 2016 / Published: 10 October 2016

A porous carbonaceous material is synthesized by direct carbonization of zeolitic imidazolate framework-67 (ZIF-67). A series of analytical tools such as scanning/transmission electron microscopy, gas chromatography/mass spectroscopy, thermogravimetric analysis, nitrogen adsorption, $\mathrm{X}$-ray diffraction, X-ray photoelectron spectroscopy and 3D tomography are conducted for the characterization of the prepared carbonaceous material (ZIF-C). ZIF-C has a well-defined concave dodecahedral shape, and its chemical composition, surface area and electrical conductivity substantially depend on carbonization temperature. ZIF-C heat-treated at $800^{\circ} \mathrm{C}$ (ZIF-C-800) shows a typical nitrogen adsorption-desorption isotherm of mesoporous materials with unimodal pores around $2 \mathrm{~nm}$ and sufficiently high electrical conductivity comparable to that of carbon nanotubes. ZIF-C-800 has Co metal particles wrapped by graphene layers on the walls of the interior open channels, and its framework is composed of Co-N, C-N species and C-C networks. ZIF-C-800 also displays the highest oxygen reduction reaction catalytic activity among ZIF-C treated at various temperatures, and its feasibility as cathode electrocatalysts for fuel cells is demonstrated by confirming the single cell performance.

Keywords: zeolitic imidazolate framework, mesoporous material, fuel cell, electrocatalyst, oxygen reduction 
(C) 2016 The Authors. Published by ESG (www.electrochemsci.org). This article is an open access article distributed under the terms and conditions of the Creative Commons Attribution license (http://creativecommons.org/licenses/by/4.0/). 